\title{
Extracorporeal membrane oxygenation for everyone?
}

\author{
J. Hunter Mehaffey, MD, MSc
}

From the Department of Surgery, University of Virginia, Charlottesville, Va.

Disclosures: Author has nothing to disclose with regard to commercial support.

Received for publication Dec 22, 2017; accepted for publication Dec 23, 2017; available ahead of print Jan 20, 2018.

Address for reprints: J. Hunter Mehaffey, MD, MSc, Department of Surgery, University of Virginia, Box 800679

Charlottesville, VA 22903 (E-mail: jhm9t@virginia.edu).

J Thorac Cardiovasc Surg 2018;155:2207-8

$0022-5223 / \$ 36.00$

Copyright (C) 2018 by The American Association for Thoracic Surgery

https://doi.org/10.1016/j.jtcvs.2017.12.099

Dr Hoetzenecker and colleagues ${ }^{1}$ are to be congratulated on an outstanding volume of clinical lung transplants with excellent outcomes. The authors propose broad application of intraoperative venoarterial extracorporeal membrane oxygenation (ECMO) for all patients undergoing lung transplantation with a low threshold for prolonged postoperative venoarterial ECMO for patients with poor initial graft function. Over the 6-year study period, the authors reviewed approximately 600 bilateral lung transplants and developed a standard protocol for use of ECMO support demonstrating superior long-term outcomes. Although this study is limited by the retrospective nature, the authors' use of propensity score matching identified 2 groups of patients, one receiving intraoperative ECMO and one receiving transplantation without ECMO, with similar baseline characteristics. In this subset, patients receiving transplantation with intraoperative ECMO required more blood transfusions but had improved outcomes and long-term survival.

Although the use of mechanical support during bilateral lung transplant is not a new idea, advancements in ECMO technology have provided significant advantages over standard cardiopulmonary bypass. ${ }^{2}$ These include a reduced inflammatory response with no venous reservoir, eliminating the air liquid interface in addition to no cardiotomy sucker, so blood is not being washed and returned. Furthermore, lower heparinization requirements with ECMO result in less postoperative coagulopathy and blood loss. However, no intervention is without risks, and this must be considered when performing additional invasive procedures in high-risk patients. The authors favor venoarterial ECMO with a central cannulation strategy intraoperatively and conversion to femoro-femoral venoarterial cannulation if postoperative ECMO is required. Although venoarterial ECMO provides significant benefits for hemodynamic stability, it is associated with higher rates of ischemic, embolic, and bleeding complications. ${ }^{3}$ The ischemic complications are particularly problematic with femoro-femoral

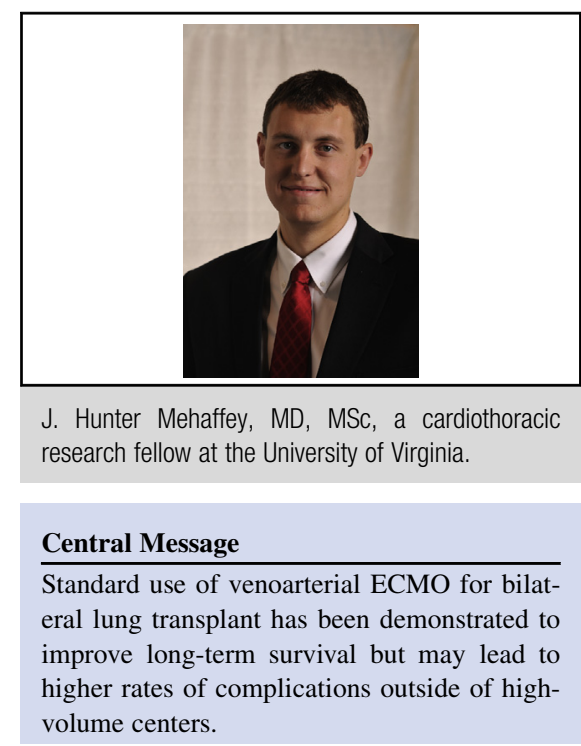

See Article page 2193.

cannulation, and most centers advocate for prophylactic placement of a distal perfusion catheter. ${ }^{4}$

This study provides provocative evidence supporting the standard use of ECMO for bilateral lung transplant. However, prospective randomized data should be assessed before widespread implementation of this practice because of increased risk of complications with additional invasive procedures, which may be compounded outside of a highvolume center. In addition, the protocol presented in the present study did not address the role of other adjuvants for lung transplant including ex vivo lung perfusion, which has gained widespread acceptance for the evaluation of lungs before transplantation. Perhaps preoperative rehabilitation and optimization of lungs with ex vivo lung perfusion would negate any benefit of intraoperative ECMO during transplantation and prevent the need for additional invasive procedures for the patient. ${ }^{5}$

\section{References}

1. Hoetzenecker K, Schwarz S, Muckenhuber M, Benazzo A, Frommlet F, Schweiger $\mathrm{T}$, et al. Intraoperative extracorporeal membrane oxygenation and the possibility of postoperative prolongation improve survival in bilateral lung transplantation. J Thorac Cardiovasc Surg. 2018;155: 2193-206.e3.

2. Magouliotis DE, Tasiopoulou VS, Svokos AA, Svokos KA, Zacharoulis D Extracorporeal membrane oxygenation versus cardiopulmonary bypass during lung transplantation: a meta-analysis. Gen Thorac Cardiovasc Surg. 2018;66: $38-47$. 
3. Napp LC, Kuhn C, Hoeper MM, Vogel-Claussen J, Haverich A, Schäfer A, et al. Cannulation strategies for percutaneous extracorporeal membrane oxygenation in adults. Clin Res Cardiol. 2016;105:283-96.

4. Lamb KM, DiMuzio PJ, Johnson A, Batista P, Moudgill N, McCullough M, et al. Arterial protocol including prophylactic distal perfusion catheter decreases limb ischemia complications in patients undergoing extracorporeal membrane oxygenation. J Vasc Surg. 2017;65:1074-9.

5. Mehaffey JH, Charles EJ, Sharma AK, Money DT, Zhao Y, Stoler MH, et al. Airway pressure release ventilation during ex vivo lung perfusion attenuates injury. J Thorac Cardiovasc Surg. 2017;153:197-204. 